\title{
DEEP LEARNING FOR IDENTIFYING MALICIOUS FIRMWARE
}

\author{
David Noever and Samantha E. Miller Noever
}

PeopleTec, Inc., 4901 Corporate Drive. NW, Huntsville, AL, USA

\begin{abstract}
A malicious firmware update may prove devastating to the embedded devices both that make up the Internet of Things (IoT) and alsothat typically lack the same security verifications now applied to full operating systems. This work converts the binary headers of 40,000 firmware examples from bytes into 1024-pixel thumbnail images to train a deep neural network. The aim is to distinguish benign and malicious variants using modern deep learning methods without needing detailed functional or forensic analysis tools. One outcome of this image conversion enables contact with the vast machine learning literature already applied to handle digit recognition (MNIST). Another result indicates that greater than $90 \%$ accurate classifications prove possible using image-based convolutional neural networks (CNN) when combined with transfer learning methods. The envisioned CNN application would intercept firmware updates before their distribution to IoT networks and score their likelihood of containing malicious variants.
\end{abstract}

\section{KEYWORDS}

Neural Networks, Internet of Things, Image Classification, Firmware, MNIST Benchmark.

\section{INTRODUCTION}

One classic benchmark for machine learning is handwriting digit recognition (Modified National Institute of Standards and Technology database, or MNIST) [1-12]. The original digit recognition challenge has since seen widespread generalization to include alphabetic versions [2] in multiple languages [10-12] and multiple unrelated topic areas [13-19] ranging across medical [13], fashion [14], and satellite imagery [17]. A common element of these generalizations has been that small images (either $28 \times 28$ or $32 \times 32$ ) $[1,7,16]$ can be addressed with both statistical machine learning (e.g. tree-based algorithms) or deep learning (multi-layer neural networks) [7]. We have recently built many cyber-security challenge datasets for malware and intrusion detection by first assembling the dataset in formats compatible with previous MNIST solutions [17-19], but also adding to the conversation begun by Intel and Microsoft Research to go beyond the signaturebased methods of identifying viruses in their STAMINA initiative [20-21]. Our datasets for malware (V-MNIST) [18] and image-based intrusion detection [19] are starting points for motivating the current approach to map firmware updates [22-23] that are either malicious, hacks, or benign into a similar format. The approach builds on the extensive publication history of mapping integer datasets to images, then applying the power of convolutional neural networks (CNNs) along with other algorithms to compare their ability to detect malicious or rogue firmware updates [24-25]. One motivation for converting the malware to imagery stems from the advanced feature extractions available for performing convolutions on pixel maps. The core mathematical transformation applied in two-dimensional convolutions includes sliding a small weight matrix over the image, performing elementwise multiplication within that particular sliding window, then finally summing up the results to generate new output pixel layers. David C. Wyld et al. (Eds): CMLA, CIoT, CSEIT, NCS, NLPD, SPM - 2021 
Successive layers involving convolutions automate feature extraction and hierarchies of related image parts. A second investigative motivation behind this approach follows from the success already demonstrated by STAMINA for other categories of malware [20-21], but extended here for firmware rather than traditional malware.

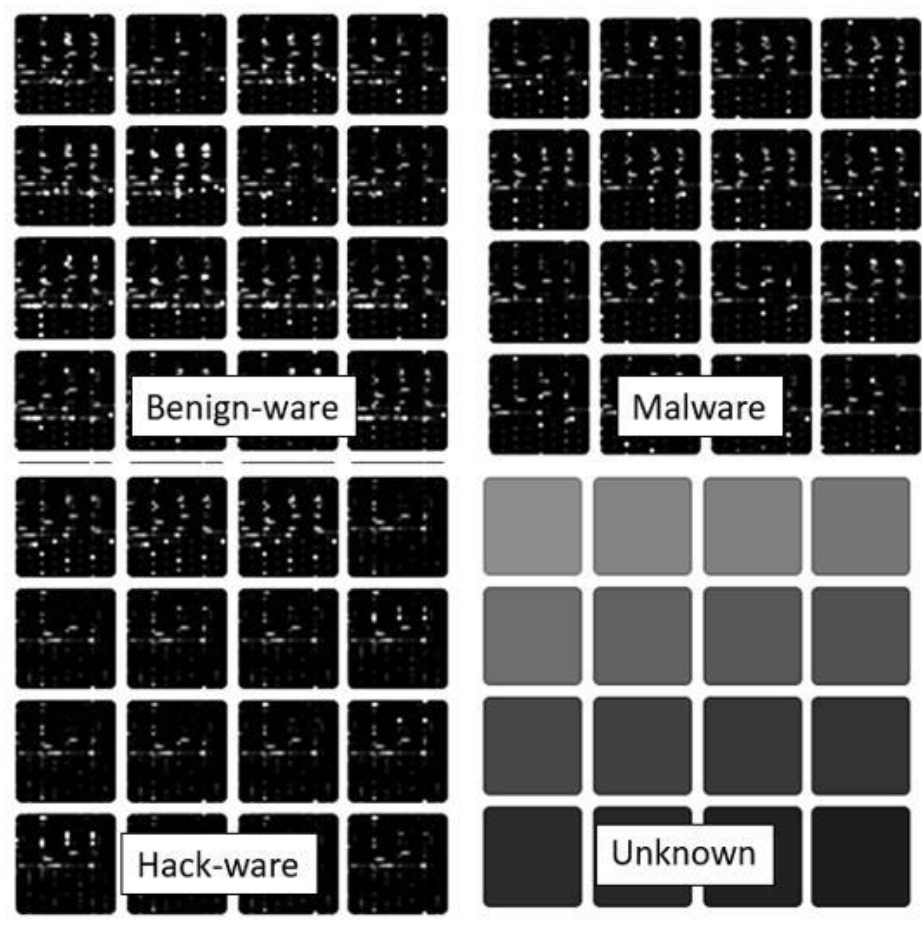

Figure 1. Firmware ELF binaries as Thumbnail Images

The future of embedded and Internet of Things (IoT) infrastructure depends on updates that users and industry can trust. What's unclear presently however is whether these updates will prove equally trustworthy given the lackadaisical approach to decent password protection or verifiable software integrity [26-27]. In 2020, 50 billion IoT devices worldwide are specifically designed to attach to a network with little or no administrative management or oversight [27]. While advanced persistent threats (APTs) have previously exploited weak passwords for devices like thermostats, home appliances, and personal assistants, the infection of firmware updates represents a larger attack surface to exploit. Anecdotal reports from the 2018 Olympics noted that hacked remote printers were unable to issue gate tickets for the opening ceremonies [27-28]. Ideally, a simple image classifier that quickly identifies and isolates rogue firmware might prove useful in the same way that program hashes and signatures defined a previous generation of malware protection layers. The original contribution of this work is to 1) map the firmware labeled dataset to a representative image and 2) solve the classification problem as a proof of principle for future development.

\section{MethodS}

This research extends the labeled ELF-binary dataset [22] to image classification. We accept the multi-class labels for malware, hack-ware, and benign-ware, which include over 40,000 examples of small compiled binaries. We add class specific to image classification which is grayscale "unknown" and bears no family resemblance to compiled software. The unknowns are just a spectrum of flattened backgrounds shades. The original dataset encodes the binary files using the following annotation and naming scheme: 
$\{$ Architecture $\} \_\{\text {Bit width }\}$ _Endianess $\} \_\{A B I\} \_\{\text {Compiler used to compile the }$ exe __\{Optimization level\}_\{Whether obfuscation was applied $\}$ _Is the file stripped of debug symbols\}_\{Package name\}__Program name\}.

\subsection{Dataset Preparation}

Employing the methods of Project STAMINA from Intel and Microsoft [21], we similarly convert the first 1024 bytes of each firmware binary to its decimal equivalent then scale those integers $(0-15)$ to span the full 0-255 interval to create small images as JPEGs. Because the class imbalances include dominant benign firmware (75\% of examples), we produced an alternative public dataset (published on Kaggle [29]) that includes both a long and a short-form version. The short-form version includes 3,000 examples of benign-ware, 714 examples of malware, and 100+ examples of hack-ware. While not balanced, it matches with the presentation of a basic confusion matrix of train-valid-test split. The choice for 1024 bytes as a small thumbnail (32x32 pixels in grayscale) derives from matching this complex problem to previous MNIST approaches but with attention to the stride-length (powers of 2) preferred by some modern deep learning frameworks like Keras. The area of the sliding weight matrix or kernel in 2D convolution determines the number of input features from the firmware that get passed to generate new output features in the deeper layers of the neural network.

\subsection{Model Parameters and Quantitative Metrics}

As an example of applying deep learning, we solve the firmware-image classifier problem using transfer learning from MobileNetV2 starting networks [30]. This network provides an optimized algorithm for feature hierarchies but efficiently extends to new areas beyond its original training datasets. We have previously found this approach useful to understand the image classification for both malware (V-MNIST) [18] and intrusion detection [19]. We use transfer learning over 50 epochs, with a 0.001 learning rate, and report four firmware classes: "malware", "hack-ware", "benign-ware", [22] and the new class labeled "unknown". The unknowns were to handle images outside of the patterns of ELF headers, such as flat grayscale backgrounds. We generate all the images using the ImageMagick tool suite [31] after binary-to-scaled decimal conversions of 1024 pixels, which subsequently rescale to meet the $32 \times 32$ requirement. The accuracy and misclassification (via error matrix) provide a score to assess effectiveness. We assess the learning parameters and sample sizes [32] using error and accuracy values per training epoch for both validation and training subsets.

\subsection{Traditional Statistical Machine Learning Approaches}

To compare the effectiveness of deep learning, we solve the tabular equivalent of the firmware in pixel format but applying tree-based methods [33]. These methods such as decision trees and random forests offer robust interpretability for why they may assign a class label to the malicious firmware. The choice between accuracy, speed, and explainability thus provides additional model tradeoffs and focuses future avenues for investigation. For example, particularly appealing output from tree-based methods includes the assignment of variable or feature importance in an automated way; among the 1024 bytes in the firmware's header, the method can extract the key positional bytes that signal a possible malicious operation. 


$\begin{array}{lrrr} & \text { benignware } & \text { hackware } & \text { malware } \\ \text { pixe1265 } & 10.07 & 11.52 & 12.81 \\ \text { pixe1633 } & 10.48 & 9.01 & 12.78 \\ \text { pixe1725 } & 7.45 & 10.43 & 12.37 \\ \text { pixe1685 } & 8.69 & 9.67 & 11.47 \\ \text { pixe1597 } & 8.19 & 7.94 & 11.13 \\ \text { pixe1781 } & 6.09 & 7.21 & 10.94 \\ \text { pixe1329 } & 11.01 & 11.21 & 10.93 \\ \text { pixe1275 } & 8.26 & 7.97 & 10.61 \\ \text { pixel653 } & 7.52 & 9.30 & 10.39 \\ \text { pixe1621 } & 8.34 & 8.80 & 10.30\end{array}$

Figure 2. Most Determinant Byte (or Pixel ) Positions for Firmware Class Assignment using Random Forest

\section{RESULTS}

\subsection{Transfer Deep Learning}

Table 1 shows the accuracy for class determinations for the small $(96 \times 96)$ and large $(224 \times 224)$ images when custom training the MobileNetV2 architecture. The choice of small images (which are rescaled from the original $32 \times 32$ ) accommodates cameras for embedded systems such as Arduino BLE Sense micro-controllers. The accuracy for a class decision approaches $100 \%$ for the larger images and suggests the ELF headers provide a sufficiently rich pattern in the first 1024 bytes to assign a risk factor to each firmware binary.

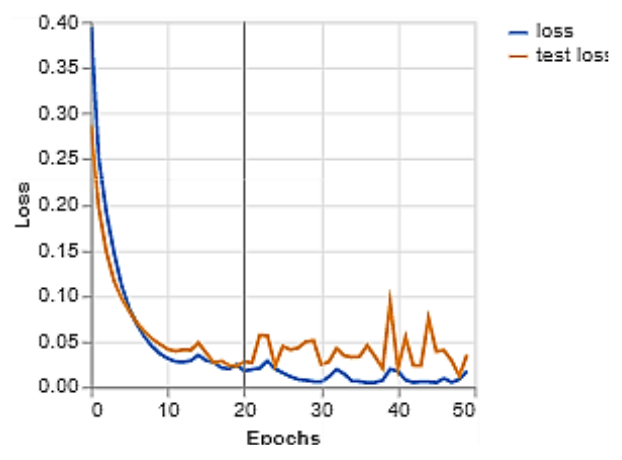

Figure 3. Learning Loss Rates over Time in Epochs

The training time versus accuracy (entropy loss) is shown in Figure 3. After 10 epochs, the network has effectively reached its plateau both for training and validation subsets. The execution times for this style of MobileNetV2 approaches real-time (equivalent to 30 frames per second), such that the overall processing for validating firmware might be limited only by the time to read the first 1024 bytes and flatten them to a decimal equivalent in pictures.

\begin{tabular}{|l|ll|ll|}
\hline \multicolumn{3}{|c|}{ Table 1. Accuracy Results for Four Class MobileNetV2 } \\
\hline Class & Lg. Accuracy (Test Samples) & $\begin{array}{l}\text { Sm. Accuracy (Test } \\
\text { Samples) }\end{array}$ \\
\hline Benign-ware & 0.98 & $(451)$ & 0.98 & $(451)$ \\
\hline Malware & 1.00 & $(107)$ & $0.94 \quad(107)$ \\
\hline Hack-ware & 1.00 & $(16)$ & $0.81 \quad(16)$ \\
\hline Unknown & $1.00 \quad(101)$ & $1.00 \quad(101)$ \\
\hline
\end{tabular}




\subsection{Single Decision Tree}

Figure 4 shows a single decision tree based on considering all 1024 pixel values but splitting firmware class determinations based on ranges of grayscale (or decimal-byte conversions) in the ELF header. Using a subset (4\%) of the full training dataset and further holding out a $15 \%$ test dataset for evaluation, the decision tree method achieves $95.9 \%$ accuracy (4.1\% error) across all three classes (benignware, hackware, and malware). This result is competitive with the deep learning approach (99+\% accuracy, Table 1). Single trees offer the additional advantage of easier interpretability. One can, for instance, envision a simple algorithm for detecting malicious firmware by examing the decimal conversion of selected key binary bytes in the ELF header. Figure 4 shows the most important 10 bytes as positions at 1285, 377, 298, and so forth. A shortcoming of this approach for single decision trees, however, stems from their brittleness, particularly when applied to test data outside of the narrow training threshold. If an attacker discovers the key 10 bytes for this method to assign a malware or hackware class to the binary, then the decision tree suffers from the same fragility as hash-based or signature methods. A single-byte change can render the detector ineffective.

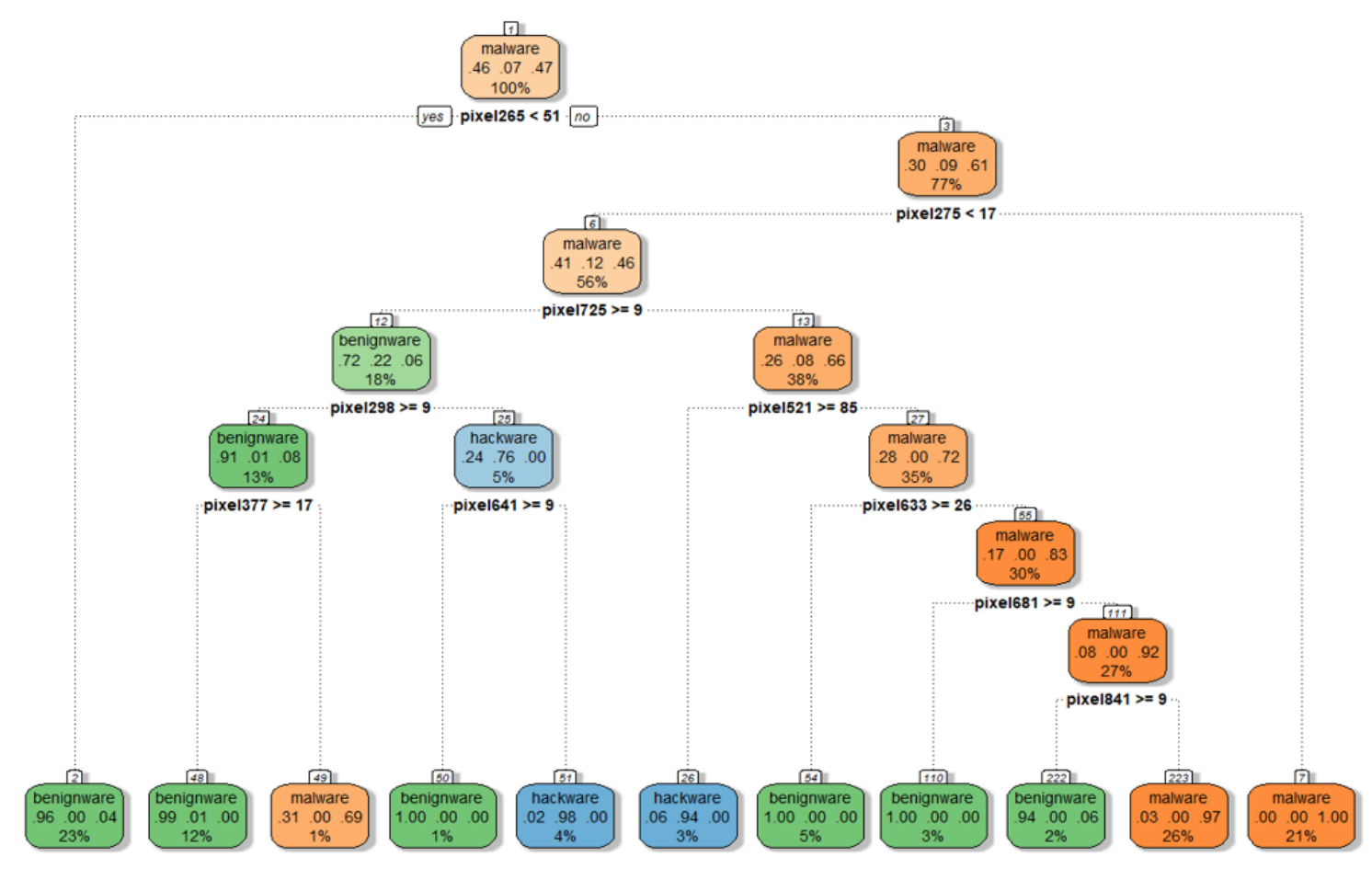

Figure 4. Single Decision Tree Applied to Firmware ELF Bytes

\subsection{Multiple Decision Trees, or Random Forest}

To investigate the robustness of statistical methods compared to deep learning, Figure 5 illustrates the application of a random forest [33]. Compared to Table 1 for CNNs, the random forest achieves 100\% class accuracy. The circular plot in Figure 5 is much denser with decision branches than the single tree shown in Figure 4. Starting in the center of the plot, decision branches for (yes-no) choices span out until a labeled class can be identified by the outer (colored) tags. The resulting high accuracy model combines an ensemble of 500 such trees to render a perfect classification for withheld testing data. The approach of combining many (often weaker) learners to render an ensembled strong learner is well-known for its enhanced robustness 
and ability to generalize better than single trees when confronted with out-of-band or underrepresented data.

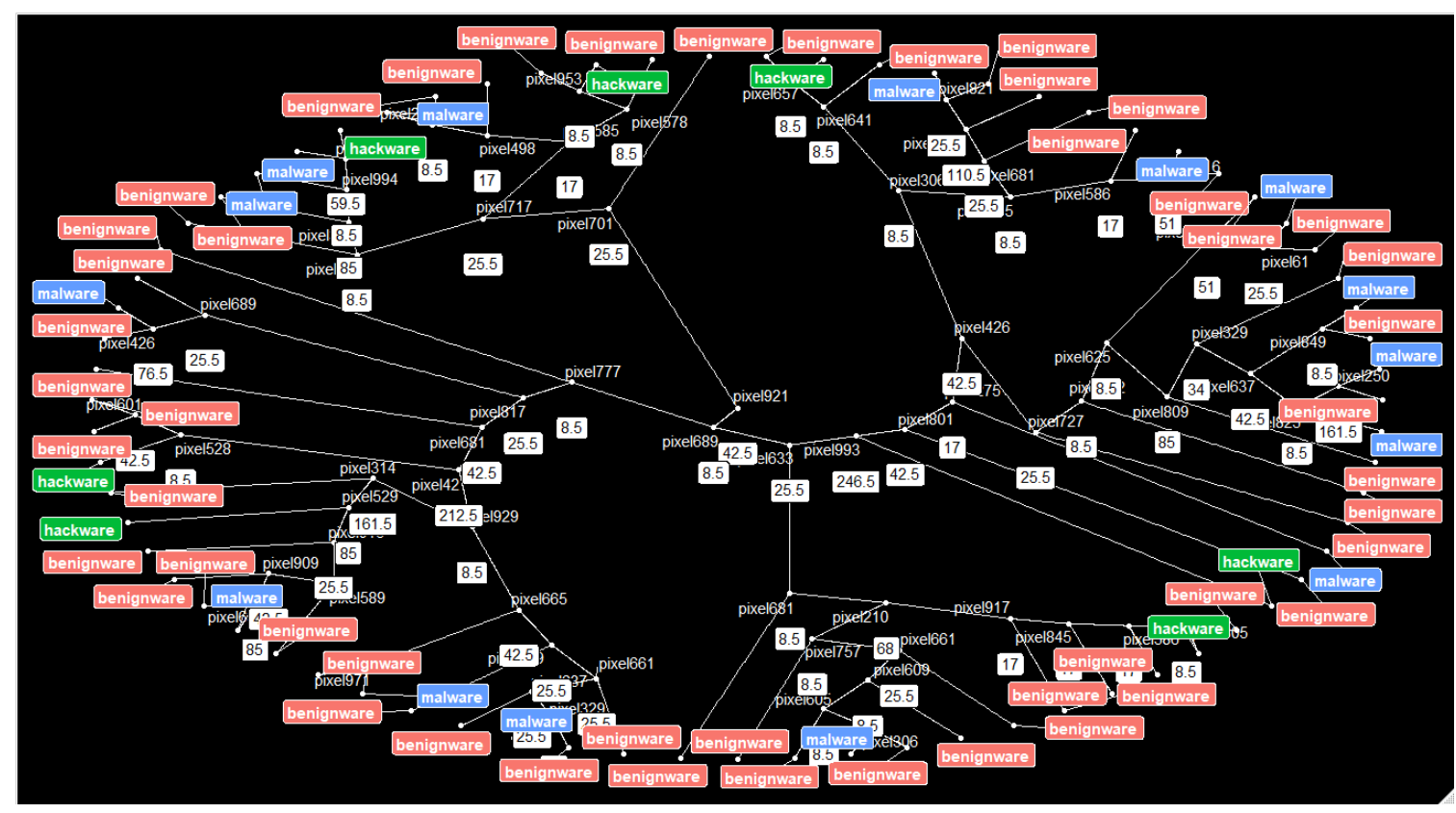

Figure 5. Random Forest (Tree 1) For Firmware Class

\section{DisCuSSION AND CONCLUSIONS}

By applying deep (transfer) learning to converted images of firmware headers, an optimized neural network can classify malicious Executable and Linkable Files (ELF). The small (32x32) grayscale images match with a decimal conversion (0-15) of the raw binary and then are scaled to a wider (0-255) pixel value range. Each pixel represents a byte in order and the underlying pattern of malicious behavior appears across the file and image nomenclature [22] for architecture, compiler, program name, etc. A procedure to under-sample the benign firmware better rebalances the dataset but leaves between 100-3000 images per class. This number of representative samples has previously been shown to be sufficient, particularly when not training the network from scratch but inherited the weighted features from a previous run on unrelated classes (transfer-learning) [32]. Future work can apply the large research efforts of MNIST derivatives to this firmware classification including simpler or more easily explainable algorithms that are tree-based methods. The research highlights an accurate tree-based method that offers additional interpretability advantages and suggests new ways to apply "if-then" filtering to ELF binaries before firmware updates.

\section{ACKNOWLEDGMENTS}

The authors would like to thank the PeopleTec Technical Fellows program for its encouragement and project assistance.

\section{REFERENCES}

[1] LeCun, Yann, Corinna Cortes, and C. J. Burges. "MNIST handwritten digit database." (2010): 18.http://yann.lecun.com/exdb/mnist/ and Y. LeCun, L. Bottou, Y. Bengio, and P. Haffner. 
"Gradient-based learning applied to document recognition." Proceedings of the IEEE, 86(11):22782324, November 1998

[2] Cohen, Gregory, Saeed Afshar, Jonathan Tapson, and Andre Van Schaik. "EMNIST: Extending MNIST to handwritten letters." In 2017 International Joint Conference on Neural Networks (IJCNN), pp. 2921-2926. IEEE, 2017.

[3] CV Online (accessed 01/2021) http://homepages.inf.ed.ac.uk/rbf/CVonline/Imagedbase.htm

[4] Google Scholar search (accessed 01/2021), https://scholar.google.com/scholar?q=mnist and https://rends.google.com/trends/explore?date=all\&q=mnist,ImageNet,\%2Fg\%2F11gfhw_78y

[5] Chen, Li, Song Wang, Wei Fan, Jun Sun, and Satoshi Naoi. "Beyond human recognition: A CNNbased framework for handwritten character recognition. "In 2015 3rd IAPR Asian Conference on Pattern Recognition (ACPR), pp. 695-699. IEEE, 2015.

[6] Image Classification on MNIST, (accessed 01/2021), https://paperswithcode.com/sota/imageclassification-on-mnist

[7] Grim, Jir1, and Petr Somol. "A Statistical Review of the MNIST Benchmark Data Problem."http://library.utia.cas.cz/separaty/2018/RO/grim-0497831.pdf

[8] Schott, Lukas, Jonas Rauber, Matthias Bethge, and Wieland Brendel. "Towards the first adversarially robust neural network model on MNIST." arXiv preprint arXiv:1805.09190 (2018).

[9] Cheng, Keyang, Rabia Tahir, LubambaKasangu Eric, and Maozhen Li. "An analysis of generative adversarial networks and variants for image synthesis on MNIST dataset." Multimedia Tools and Applications 79, no. 19 (2020): 13725-13752.

[10] Preda, Gabriel, Chinese MNIST: Chinese Numbers Handwritten Characters Images, (accessed 01/2021) https://www.kaggle.com/gpreda/chinese-mnist

[11] CoMNIST: Cyrillic-oriented MNIST, A Dataset of Latin and Cyrillic Letters, (accessed 01/2021) https://www.kaggle.com/gregvial/comnist

[12] Prabhu, Vinay Uday. "Kannada-MNIST: A new handwritten digits dataset for the Kannada language." arXiv preprint arXiv:1908.01242 (2019).https://www.kaggle.com/higgstachyon/kannadamnist

[13] Noever, David, Noever, Sam E.M. "Expressive Multimodal Integrated Learning (EMIL): A New Dataset for Multi-Sense Integration and Training”, 2020 Southern Data Science Conference, August 12-14 2020, Atlanta, GA (poster) and Sign Language MNIST: Drop-In Replacement for MNIST for Hand Gesture Recognition Tasks, https://www.kaggle.com/datamunge/sign-language-mnist

[14] Mader, K Scott, Skin Cancer MNIST: HAM 10000, A Large Collection of Multi-Source Dermatoscopic Images of Pigmented Lesions, (accessed 01/2021) https://www.kaggle.com/kmader/skin-cancer-mnist-ham 10000

[15] Xiao, Han, Kashif Rasul, and Roland Vollgraf. "Fashion-MNIST: a novel image dataset for benchmarking machine learning algorithms." arXiv preprint arXiv: 1708.07747 (2017).https://www.kaggle.com/zalando-research/fashionmnistSee also FashionMNIST, (accessed 01/2021), https://paperswithcode.com/sota/image-classification-on-fashion-mnist and https://github.com/zalandoresearch/fashion-mnist

[16] Lu, Arlen, "Convert-own-data-to-MNIST-format" $\quad$ (accessed 01/2021) https://github.com/Arlen0615/Convert-own-data-to-MNIST-format

[17] Noever, D., \& Noever, S. E. M. (2021). Overhead MNIST: A benchmark satellite dataset. arXiv preprint arXiv:2102.04266.https://www.kaggle.com/datamunge/overheadmnist and Github, https://github.com/reveondivad/ov-mnist

[18] Noever, D., \& Noever, S. E. M. (2021). Virus-MNIST: A Benchmark Malware Dataset. arXiv preprint arXiv:2103.00602.

[19] Noever, D. A., \& Noever, S. E. M. (2021). Image Classifiers for Network Intrusions. arXiv preprint arXiv:2103.07765.

[20] Freitas, S., Duggal, R., \& Chau, D. H. (2021). MalNet: A Large-Scale Cybersecurity Image Database of Malicious Software. arXiv preprint arXiv:2102.01072

[21] Chen, L., Sahita, R., Parikh, J., Marino, M. (2020). STAMINA: Scalable Deep Learning Approach for Malware Classification, https://www.intel.com/content/dam/www/public/us/en/ai/documents/stamina-scalable-deep-learningwhitepaper.pdf

[22] Partush, N. (2021). Labeled-Elfs, https://github.com/nimrodpar/Labeled-Elfs 
[23] Kairajärvi, S., Costin, A., \&Hämäläinen, T. (2019). Towards usable automated detection of CPUarchitecture and endianness for arbitrary binary files and object code sequences. arXiv preprint arXiv:1908.05459.

[24] Clemens, J. (2015). Automatic classification of object code using machine learning. Digital Investigation, 14, S156-S162.

[25] Xie, H., Abdullah, A., \&Sulaiman, R. (2013). Byte frequency analysis descriptor with spatial information for file fragment classification. In Proceeding of the International Conference on Artificial Intelligence in Computer Science and ICT (AICS 2013).

[26] Constantin, L. (2015). Cisco warns customers about attacks installing rogue firmware on networking gear, Network World. Aug 10, 2015. https://www.networkworld.com/article/2970954/cisco-warnscustomers-about-attacks-installing-rogue-firmware-on-networking-gear.html

[27] Microsoft Threat Intelligence Center(2019). Corporate IoT - a path to intrusion. https://msrcblog.microsoft.com/2019/08/05/corporate-iot-a-path-to-intrusion/

[28] Greenberg, A. (2019). The Untold Story of the 2018 Olympics Cyberattack, the Most Deceptive Hack in History. Wired Magazine. https://www.wired.com/story/untold-story-2018-olympics-destroyercyberattack/

[29] Noever, D. (2021). IoT Firmware Image Classifier: Rendered ELF Binaries by Class as Malware, Kaggle. https://www.kaggle.com/datamunge/iot-firmware-image-classification

[30] Sandler, Mark, Andrew Howard, Menglong Zhu, Andrey Zhmoginov, and Liang-Chieh Chen. "Mobilenetv2: Inverted residuals and linear bottlenecks." In Proceedings of the IEEE conference on computer vision and pattern recognition, pp. 4510-4520. 2018.

[31] Salehi, Sohail. ImageMagick Tricks. Packt publishing ltd, 2006.

[32] Warden, P. "How many images do you need to train a neural network?" (2017).https://petewarden.com/2017/12/14/how-many-images-do-you-need-to-train-a-neuralnetwork/

[33] Morales-Molina, C. D., Santamaria-Guerrero, D., Sanchez-Perez, G., Perez-Meana, H., \& HernandezSuarez, A. (2018, November). Methodology for malware classification using a random forest classifier. In 2018 IEEE International Autumn Meeting on Power, Electronics and Computing (ROPEC) (pp. 1-6). IEEE.

(C) 2021 By AIRCC Publishing Corporation. This article is published under the Creative Commons Attribution (CC BY) license. 